\title{
Acute disseminated encephalomyelitis after severe acute respiratory syndrome coronavirus 2 vaccination: a case report
}

\author{
Liming Cao ${ }^{1,2}\left(\right.$ Lijie $^{1,3}$
}

Received: 4 December 2020 / Accepted: 18 January 2021 / Published online: 1 February 2021

(c) Belgian Neurological Society 2021

Keywords Severe acute respiratory syndrome coronavirus 2 Vaccination - Acute disseminated encephalomyelitis . Gammaglobulin

\section{Dear Editor:}

The severe acute respiratory syndrome coronavirus 2 (SARS-CoV-2) infection outbreak is a global pandemic defined by the World Health Organization, and vaccines are well into development. Vaccine safety is not described, and severe neurological complications may occur [1]. Acute disseminated encephalomyelitis (ADEM) is an immunemediated demyelinating disease with diverse clinical manifestations, extensive central nervous system white matter involvement, and multiple risk factors, including vaccines $[2,3]$. ADEM generally has a favorable outcome.

A 24-year-old woman was admitted after a 1-day memory decline with headache, low-grade fever, muscle stiffness, extremity weakness, and reduced appetite. She reported no upper respiratory infection or diarrhea. Two weeks prior, she received a SARS-CoV-2 vaccine, including $4 \mu \mathrm{g}$ inactivated SARS-CoV-2 (Vero Cells, Beijing Institute of Biological Products Co., Ltd., Beijing, China). She has no significant medical history. Neurological examination showed somnolence and memory decline, although other neurological tests were within normal limits. Her Mini-Mental State Examination (MMSE) score was 11 out of 30.

Upon hospitalization, the cerebrospinal fluid (CSF) white blood cell (WBC) count was $51 \times 10^{6} / \mathrm{L}$ (reference range, $0-10 \times 10^{6} / \mathrm{L}$ ). Two days later, a repeat CSF examination showed a WBC count of $25 \times 10^{6} / \mathrm{L}$. CSF was negative for

Liming Cao

caolm-2007@163.com

1 Department of Neurology, Shenzhen University First Affiliated Hospital, Shenzhen, China

2 Department of Neurology, The Third Affiliated Hospital of Shenzhen University, Shenzhen, China

3 Department of Neurology, Shenzhen Second People's Hospital, Shenzhen, China antibodies to major pathogens and cultures of bacteria and fungi; high-throughput genome sequencing also revealed no pathogens. SARS-CoV-2 antibodies were negative in the CSF on day 1 and in her blood on day 2 after admission. SARS-CoV-2 immunoglobulin $\mathrm{G}$ was present in the blood on day 5 after admission. Oligoclonal bands in blood and CSF were negative, and CSF antibodies, including anti-aquaporin-4, anti-myelin basic protein, anti-myelin oligodendrocyte glycoprotein, anti-glial fibrillary acidic protein, autoimmune encephalitis, and paraneoplastic syndrome, were negative. Six nasal swab tests for SARS-CoV-2 nucleic acid amplification performed at different times during hospitalization were negative. HIV, autoimmune vasculitis, anticardiolipin, and anti-nuclear antibody tests of blood were all negative.

Initial brain magnetic resonance imaging (MRI) showed abnormal signals in the bilateral temporal cortex (Fig. 1a-h). Repeat brain MRI on day 10 showed an increased number of lesions, which were more striking in appearance (Fig. 1i-1); the lesions were improved by day 15 (Fig. $1 \mathrm{~m}-\mathrm{p}$ ). The patient was administered intravenous (IV) ceftriaxone $2 \mathrm{~g} /$ days for 12 days and acyclovir $1.5 \mathrm{~g} /$ days for 16 days. Her headache, low-grade fever, and muscle aches were relieved while undergoing during treatment, although her poor memory persisted. She had a generalized tonic-clonic seizure 1 week after admission. An electroencephalogram showed epileptiform waves. She was immediately administered IV diazepam $10 \mathrm{mg}$ and oral levetiracetam $1 \mathrm{~g} /$ days, with no seizure recurrence. She was administered IV immunoglobulin $20 \mathrm{~g}$ /days for 5 days starting on day 11 , and her MMSE scores improved to 29/30 on day 15. On a visit 1 month after discharge, she felt no discomfort, and a repeat MRI showed complete resolution of brain lesions.

Our patient met the diagnostic criteria set by the International Pediatric MS Study Group [4], and alternative 


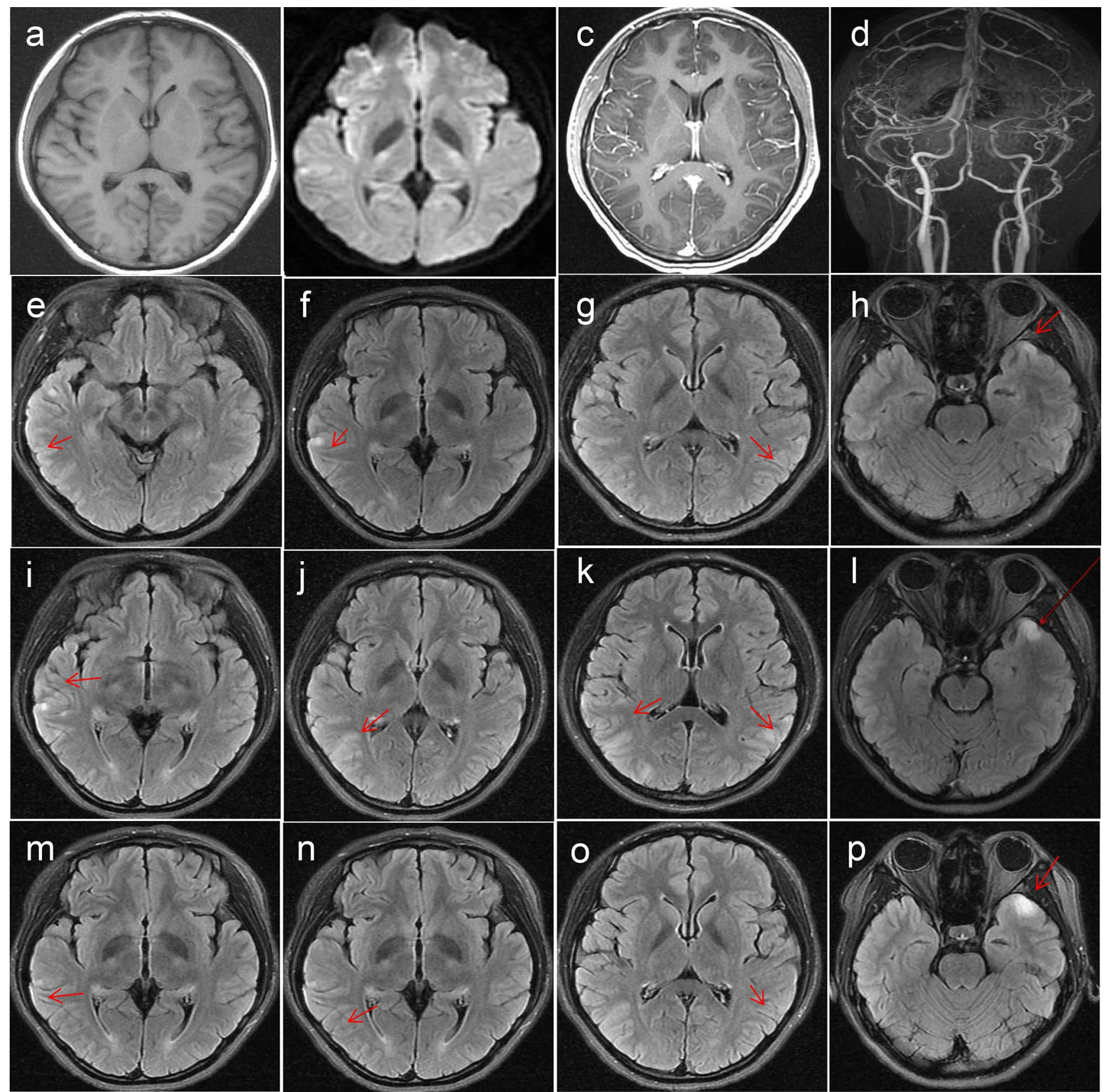

Fig. 1 MRI studies during hospitalization. Day 2 after hospitalization: T2-weighted imaging (WI; a arrow), diffusion-WI (b arrow), contrast-enhanced MRI (c), and MR angiography (d) show no obvious abnormality. Fluid-attenuated inversion recovery (FLAIR, e-h, arrows) imaging shows hyperintense signals, while T1-WI (e) shows

diagnoses, such as infectious or autoimmune encephalitis, were excluded in our patient. ADEM following vaccination is a well-known entity and is, hence, not surprising following SARS-CoV-2 vaccination. In this case, a protective antibody was produced after vaccination. MRI typically shows reversible, large $(>1-2 \mathrm{~cm})$, bilateral, white matter lesions, and deep gray matter lesions of the brain. CSF analysis may low signals in the bilateral temporal cortices. Day 10 after hospitalization: repeat FLAIR imaging (i-l, arrows) reveals an increase in the number of lesions and their more striking appearance. Day 15 after hospitalization: repeat FLAIR imaging (m-p, arrows) shows an improvement in the lesions

reveal a mild pleocytosis and elevated protein levels, but it is generally negative for intrathecal oligoclonal immunoglobulin $\mathrm{G}$ synthesis [2]. Systemic corticosteroids are firstline therapy [5], and patients have an excellent response to gamma-globulin [6].

A brief literature review did not identify a case similar to ours. ADEM has not been reported following SARS-CoV-2 
vaccination, although it is a possibility. Patients with CNS findings should be enquired about recent vaccinations for a quick and accurate diagnosis to initiate treatment and prevent serious sequelae.

Author contributions LC: writing and conceptualization; LR: diagnosis confirmation and review of MRI.

Funding This study was supported by Natural Science Foundation of Liaoning Province of China (No. 2019-MS-200).

Availability of data and material Not applicable.

\section{Compliance with ethical standards}

Conflicts of interest The authors declare that there is no conflict of interest.

Ethics approval The study design was approved by the ethics review board of the 3rd Affiliated Hospital of Shenzhen University (No: 2021-LHYYKY-LW-001).

Informed consent Informed consent was obtained from the patient included in the case report.

Consent for publication The patient provided written, informed consent for the publication of this case.

\section{References}

1. Huynh W, Cordato DJ, Kehdi E, Masters LT, Dedousis C (2008) Post-vaccination encephalomyelitis: literature review and illustrative case. J Clin Neurosci 15:1315-1322. https://doi.org/10.1016/j. jocn.2008.05.002

2. Pohl D, Alper G, Haren KV et al (2016) Acute disseminated encephalomyelitis: updates on an inflammatory CNS syndrome. Neurology 87:S38-45. https://doi.org/10.1212/WNL.0000000000 002825

3. Torisu H, Okada K (2019) Vaccination-associated acute disseminated encephalomyelitis. Vaccine 37:1126-1129. https://doi. org/10.1016/j.vaccine.2019.01.021

4. Krupp LB, Banwell B, Tenembaum S, International Pediatric MS Study Group (2007) Consensus definitions proposed for pediatric multiple sclerosis and related disorders. Neurology 68:S7-12. https://doi.org/10.1212/01.wnl.0000259422.44235.a8

5. Gray MP, Gorelick MH (2016) Acute disseminated encephalomyelitis. Pediatr Emer Care 32:395-400. https://doi.org/10.1097/ PEC.00000000000008250

6. Sonneville R, Demeret S, Klein I et al (2008) Acute disseminated encephalomyelitis in the intensive care unit: clinical features and outcome of 20 adults. Intensive Care Med 34:528-532. https:// doi.org/10.1007/s00134-007-0926-2

Publisher's Note Springer Nature remains neutral with regard to jurisdictional claims in published maps and institutional affiliations. 